\title{
Pressure-assisted microsyringe 3D printing of oral films based on pullulan and hydroxypropyl methylcellulose
}

\author{
M. Elbadawi ${ }^{1,2}$, D. Nikjoo ${ }^{3}$, T. Gustafsson ${ }^{1}$, S. Gaisford $^{2}$, and A.W. Basit ${ }^{2}$ \\ ${ }^{1}$ Control Engineering Group, Department of Computer Science, Electrical and Space \\ Engineering, Luleå University of Technology, Luleå, Sweden \\ ${ }^{2}$ Department of Pharmaceutics, UCL School of Pharmacy, University College London, 29-39 \\ Brunswick Square, London WC1N 1AX, UK. \\ ${ }^{3}$ Division of Material Science, Luleå University of Technology, Luleå, Sweden
}

\begin{abstract}
Oral films (OFs) continue to attract attention as drug delivery systems, particularly for pedatric and geriatric needs. However, immiscibility between different polymers limits the full potential of OFs from being explored. One example is pullulan (PUL), a novel biopolymer which often has to be blended with other polymers to reduce cost and alter its mechanical properties. In this study, the state-of-the-art in fabrication techniques, three-dimensional (3D) printing was used to produce hybrid film structures of PUL and hydroxypropyl methylcellulose (HPMC), which were loaded with caffeine as a model drug. 3D printing was used to control the spatial deposition of films. HPMC was found to increase the mean mechanical properties of PUL films, where the tensile strength, elastic modulus and elongation break increased from 8.9 to $14.5 \mathrm{MPa}, 1.17$ to $1.56 \mathrm{GPa}$ and from $1.48 \%$ to $1.77 \%$, respectively. In addition, the spatial orientation of the hybrid films was also explored to determine which orientation could maximize the mechanical properties of the hybrid films. The results revealed that 3D printing could modify the mechanical properties of PUL whilst circumventing the issues associated with immiscibility.
\end{abstract}

Key words: 3D printing; 3D printed drug products; Printing medicines and pharmaceuticals; Pressure Assisted Microsyringe; Oral drug delivery films; Rheology. 


\section{Introduction}

Oral films (OFs) are relatively new dosage forms that deliver drugs via the oral cavity or oromucosal route. They have garnered recent attention for use in children, geriatrics and those with either physiological or psychological induced dysphagia (Slavkova and Breitkreutz, 2015). It has been estimated that $28 \%$ of the population experiences frequent problems with swallowing medicines (Borges et al., 2015a). OF popularity as drug delivery systems (DDS) is further highlighted by high patient compliance, rapid onset of action, ease of transportation and handling, and avoidance of first-pass metabolism (Karki et al., 2016a; Silva et al., 2015), wherein more of the active pharmaceutical ingredient (API) reaches circulation. Currently, 52\% of the market share is held by products aimed at oral delivery (Borges et al., 2015a).

Pullulan (PUL) is an exopolysaccharide polymer that is garnering attention for OFs, due to its biocompatibility, biodegradability and non-mutagenic, non-carcinogenic and immunoneutrality properties (Prajapati et al., 2013; Tabasum et al., 2018a). Furthermore, as a biopolymer, PUL is abundant, biodegradable and sustainable for the environment (Cristescu et al., 2004; McKeen, 2017). Recently, the suitability of PUL for OFs has been explored because of its excellent film-forming properties (Prajapati et al., 2018; Shingel, 2004; Sugumaran and Ponnusami, 2017),

and it has demonstrated considerable mechanical strength and water-solubility, as well as being odorless and tasteless (Sathya et al., 2018). Moreover, PUL based films exhibit fast disintegration times (Borges et al., 2015b; Prajapati et al., 2018; Vuddanda et al., 2017). Despite its remarkable versatility and unique characteristics, the shortcomings of PUL are its brittleness and high cost. The traditional approach to addressing these drawbacks is through either blending or copolymerization (Kanmani and Lim, 2013; Tabasum et al., 2018b). The appeal of blending is that it offers a low-cost alternative to the development of an entirely new polymer with improved properties. However, attaining homogenous polymer blends with desirable properties is relatively unusual (Prasad et al., 2008). Copolymerisation involves drawbacks like the requirements for chemical expertise and bulk modifications (Hadal and Misra, 2005).

An alternative approach to combining polymers is to use dual-nozzle pressure-assisted microsyringe (PAM) three-dimensional printing (3DP). PAM is a subclass of 3DP, also referred to as solid freeform fabrication (SFF), that is a rapidly emerging fabrication technology(RoblesMartinez et al., 2019). The process entails designing an object with computer-aided design (CAD) 
software and transferring the model to the printer, via slicing software, for fabrication (Awad et al., 2020a; Elbadawi et al., 2020; Trenfield et al., 2020; Xu et al., 2020). An advantage of 3DP is its high spatial resolution that allows for personalized dosages to be obtained, as well as achieving complex geometries with digital precision (Awad et al., 2020b; Goyanes et al., 2015; RoblesMartinez et al., 2019; Trenfield et al., 2019; Vivero-Lopez et al., 2021; Xu et al., 2021). Utilising the dual-nozzle capabilities allows the controlled composition of polymers in a blend, thereby obtaining a structured film. The aforementioned features of PAM make it an ideal candidate for fabricating blends of polymers that are immiscible. Further advantages specific to OFs include rapid fabrication, and near-net shape fabrication, and thus minimal post-processing (Karki et al., 2016b; Scarpa et al., 2017).

The present study details the use of PAM to fabricate a blended film of PUL with a polymer that exhibits limited miscibility. As a proof of concept, hydroxypropyl methylcellulose (HPMC) was used as the secondary polymer, with caffeine as the model drug. HPMC has been blended with PUL to improve the latter's properties, but the polymers were reported to exhibit limited miscibility (Prasad et al., 2008). This study also investigated the ideal printing orientation to maximize mechanical performance when blending two polymers, because mechanical properties are a key criterion for OF usability (Preis et al., 2013).

\section{Experimental Procedure}

\subsection{Materials and Formulations}

Pullulan (PUL) (Product number AB 141364, abcr GmbH \& Co. Kg, Karlsruhe, Germany) and hydroxypropyl methylcellulose (HPMC) (PHARMCOAT 606, Shin-Etsu, Tokyo, Japan; MW: 10,000 g/mol, methoxy: 29\%, hydroxypropoxy: 9\%) biopolymers were mixed with anhydrous caffeine (Fluka, a subsidiary of Sigma Aldrich, Stockholm, Sweden), propylene glycol (High Purity Grade, AMRESCO 0575-1L, Fisher Scientific, Göteborg, Sweden) as a plasticizer, and distilled water to form polymer solutions. First, caffeine and water were added to a beaker and mixed at 70 ${ }^{\circ} \mathrm{C}$ using a magnetic stirrer and hot plate. The beaker was sealed with aluminium foil to minimize partial evaporation. Mixing was performed until caffeine, which is poorly soluble in water at room temperature, was dissolved. Subsequently, the admixture was poured into a separate beaker, whereafter weighed propylene was added and mixed using a spatula. The final step was the addition 
of the polymer, which was added in 3-5 tranches and mixed using a spatula after each tranche. Preliminary formulations (data not included) were evaluated until an extrudable solution that subsequently did not spread, was obtained. This leads to the compositions of the formulation used herein, which are enumerated in Table 1. A HPMC-PUL blended solution was also formulated for comparison, comprising the same proportion as solutions $\mathrm{H} 4$ and $\mathrm{P} 4$ except for the polymer content consisted of $50 \%$ of each biopolymer.

\subsection{Fabrication of Polymer Films}

Films were fabricated using a dual-extrusion, pressure-assisted microsyringe (Inkredible, Cellink, Gothenburg, Sweden). Once formulated, the solutions were stored at room temperature for 5 hours to allow the bubbles to rise to the surface, after which they were poured into a syringe with a $22 \mathrm{G}$ nozzle and subsequently transferred to the printer. Printing was performed on $9 \mathrm{~mm}$ glass-petri dishes, and three prints were performed simultaneously. The printing pressure was determined by setting the pressure to $0 \mathrm{kPa}$ and turning the valve until a consistent flow was attained. The samples were thereafter transferred to a desiccator and allowed to dry for three days before analysing (Figure 1). Rectangular CAD models were designed (Onshape, MA, USA) and sliced (HeartWare v2.4.1, Cellink, Gothenburg, Sweden) before being uploaded onto the printer. The slicing software allowed for pre-determining the printhead operation. For whole films, only one printhead was used to achieve 30 by $10 \mathrm{~mm}$ prints. For the bimodal printing, each half of the print was made with a separate printhead. A schematic depicting the printing orientation and dimensions of the films is included in the supplementary document (Figure S1).

\subsection{Characterisation Techniques}

\subsubsection{Rheological Characterisations of Formulations}

A rotational rheometer (DHR2, TA Instruments, DE, USA) was used for rheological analyses. All analyses were performed at ambient temperature, with the peltier plate temperature set to $23{ }^{\circ} \mathrm{C}$. Steady-state viscosity measurements were performed using a $20 \mathrm{~mm}$ cone plate $\left(2^{\circ}, 0.052\right.$ truncation gap) with a geometric gap of $1 \mathrm{~mm}$; and performed for three minutes. Oscillatory rheological analyses were performed using an $8 \mathrm{~mm}$ parallel plate, with a geometric gap of $0.5 \mathrm{~mm}$. 
Oscillation amplitude sweeps were performed at an angular frequency of $10 \mathrm{rad} / \mathrm{s}$, with an oscillatory strain range of either 0.1 to $1000 \%$. To measure the rheological stability of the solutions as a function of time, time sweeps were conducted for five minutes at a strain rate of $1 \%$ and angular frequency of $10 \mathrm{rad} / \mathrm{s}$ for all samples. For measuring the recoverable characteristics of the solutions, an oscillatory time sweep was again employed, whereby an initial small amplitude oscillatory shear (SAOS) was applied, followed by a large amplitude oscillatory shear (LAOS), and concluded with a final SAOS. The angular frequency was kept constant at $10 \mathrm{rad} / \mathrm{s}$ throughout the time sweep, with the strain rate varying from $1 \%$ for 45 seconds, to $1000 \%$ for 45 seconds, and back to $1 \%$ for 180 seconds.

\subsubsection{Fourier transform infrared (FTIR) spectroscopy.}

The characterization of the 3D printed PUL and HPMC based films was first performed by an attenuated total reflectance Fourier Transform-Infra Red (ATR-FTIR) spectroscopic technique within the range 500-4500 $\mathrm{cm}^{-1}$ using Bruker VERTEX 80v FTIR spectrometer (Ettlingen, Germany) with a resolution of $4 \mathrm{~cm}^{-1}$ with 128 scans.

\subsubsection{X-ray diffraction (XRD) analysis.}

The XRD patterns of CAF loaded PUL and HPMC films were collected by a PANalytical Empyrean instrument (Almelo, The Netherlands) with a monochromatic $\mathrm{Cu}-\mathrm{K} \alpha$ radiation $\mathrm{X}$-Ray tube $(\lambda=1.54056 \AA)$ connected to the PIXcel3D detector. The films were analyzed in the $2 \theta$ between $5^{\circ}$ and $40^{\circ}$ increasing at a step size of 0.02 with current and voltage of $40 \mathrm{~mA}$ and $45 \mathrm{kV}$, respectively. The XRD profiles were obtained at $25 \pm 1{ }^{\circ} \mathrm{C}$ and analyzed using High Score Plus software version 3.0.1.

\subsubsection{Scanning electron microscopy (SEM).}

SEM was utilized to achieve morphological analysis of HPMC and PUL based films. The films were analyzed by the microscope (JEOL JSM 6064LV, USA) after the specimens were placed on a metal plate using Leit-C conductive carbon (Plano GmbH, Wetzlar, Germany).

\subsubsection{Tensile Properties Characterisation of Polymer Films}

Tensile properties were measured using the DHR2 equipped with the linear film tension accessory (TA Instrument, DE, USA) and operated axially. The test motor was set to tension mode, with a length loading gap of $20 \mathrm{~mm}$, at a constant linear rate of $1 \mathrm{~mm} / \mathrm{s}$. The run was terminated shortly 
after the complete fracture of the sample. Films $(30$ x $10 \mathrm{~mm})$, as confirmed by a Vernier caliper, were used for mechanical analysis. A schematic depicting the tensile test setup for the hybrid films can be found in the supplementary document (Figure S2).

\subsubsection{Disintegration Time}

The modified petri dish method was used to determine the time of disintegration of the biofilms. 1 x $1 \mathrm{~cm}$ films were tested and were used as-printed. First, a $7 \mathrm{~cm}$ dish with $7 \mathrm{~mL}$ of distilled water was placed in a laboratory shaker, which was rotating at $100 \mathrm{rpm}$ and allowed to reach a temperature of $37^{\circ} \mathrm{C}$. This was allowed to continue for five minutes to ensure the petri dish and the distilled water within to reach the desired temperature. The film was then placed in the centre of the dish and the test was commenced. Time until the film began to disintegrate was recorded (Mazumder et al., 2017).

\section{Results}

The purpose of this study was to use PAM to produce hybrid film structures whilst circumventing the issue of blending immiscible polymers. Initially, three formulations were printed, which were the neat polymer solution $(\mathrm{P} 0 / \mathrm{H} 0)$, with $3 \% \mathrm{w} / \mathrm{w}$ caffeine $(\mathrm{P} 1 / \mathrm{H} 1)$ and with $10 \% \mathrm{w} / \mathrm{w}$ caffeine $(\mathrm{P} 2 / \mathrm{H} 2)$. For the latter, high printing pressures were needed, which for P2 approached the limitations of the PAM printer. Therefore, two further formulations were subsequently prepared, where the water content was increased by 3 and $6 \% \mathrm{w} / \mathrm{w}$ to reduce their viscosity, and consequently the printing pressure. In this section, the results of the characterisation are detailed for the feedstock

first, and followed by the results for the final product. For the feedstock (i.e. the solutions), different rheological characterisation techniques were performed, as rheology is a useful indicator to a material's printing performance (Elbadawi, 2019). For the final product (i.e. the films), they were characterized for their chemical structure, morphology, mechanical properties and disintegration.

\subsection{Viscosity measurements of formulations}

Viscosity measurements were performed to determine the ideal profiles for printing. All formulated solutions presented with shear-thinning characteristics (Figure 2), which is the ideal rheological behavior for extrusion-based fabrication techniques (Elbadawi, 2018). The viscosity profiles were fitted with the Cross model (Equation S1) to quantify the shear-thinning behavior. Both P4 and H4 were found to be the most shear-thinning for their respective polymer solutions. However, it 
was the blended PUL-HPMC solution that possessed the highest shear-thinning exponent. The quantified shear-thinning exponent can be found in Table S1.

The viscosity profile revealed a trend with the addition of caffeine. For PUL solutions, increasing caffeine content increased the viscosity at low shear rates, and hence a higher Newtonian plateau was seen, particularly for P2. Conversely, at higher shear rates the caffeine-free sample was found to possess the highest viscosity. With regards to varying the water content at $10 \% \mathrm{w} /$ caffeine (P2, P3, and P4), increasing the amount did not affect the viscosity at low shear rates, however, the highest water content sample (P4) was found to be more shear-thinning. Thus, increasing the water content from $60 \% \mathrm{w} / \mathrm{w}$ to $66 \% \mathrm{w} / \mathrm{w}$ affected the viscosity at high shear rates. Increasing the caffeine content from $0 \% \mathrm{w} / \mathrm{w}(\mathrm{H} 0)$ to $10 \% \mathrm{w} / \mathrm{w}(\mathrm{H} 2)$ in the HPMC solutions also increased the viscosity at low shear rates, but the viscosities were comparable by the end of the test (Figure 2 (b)). In contrast to the pullulan solutions, increasing the water content of $10 \% \mathrm{w} / \mathrm{w}$ caffeine HPMC solutions decreased the viscosity at both low and high shear rates. The PUL-HPMC (50/50) blended solution was also analysed (Figure 2 (c)), and was discovered to possess an initial viscosity in the order of $10^{4} \mathrm{~Pa}$.s, which was two orders of magnitude greater compared with pure PUL and pure HPMC solutions. Oscillatory amplitude sweeps were also performed for further insight into the rheological changes with the addition of caffeine (Figure S3). The amplitude sweeps revealed that the addition of caffeine broke down the network structures of both polymers, which were found to yield at lower strains with increasing caffeine. In addition, to ensure that the rheological changes were as a function of increasing shear rate, time ramps were also performed on all samples. The time ramps were performed for five minutes, which is a greater than both the time needed to perform the viscosity measurements, as well as the printing process. The results revealed that all solutions were rheologically stable for five minutes, hence there was no timedependent influence on either the printing or viscosity measurements (Figure S4).

\subsection{Solution Recovery}

The challenge with PAM is that a low viscous solution is desired for it to flow through nozzle sizes with diameters in the micron region. However, a low viscous solution tends to spread once printed, and thereby resulting in a deformed film print. Hence, the potential structural recovery of the solutions was also examined to elucidate the effect of caffeine on the ability of the polymers to 
recover following large deformation. Essentially, the test replicated the shearing behavior observed

during PAM; solutions were subjected to large deformations during extrusion, whereas both before and subsequently after, the admixture was subjected to small deformations. This was simulated rheologically by applying an initial small amplitude oscillatory shear (SAOS), followed by a large amplitude oscillatory shear (LAOS), and a final SAOS thereafter to observe the recovery of the solutions. Figure 3 (a) is a representative plot of the process. For all the formulated solutions, the LAOS perturbed the polymeric network to the extent where the storage modulus decreased noticeably. However, once this was removed the storage modulus was observed to recover. The data were compared to a time ramp where the strain was constantly at 1\%. (i.e. continuous SAOS; represented by the dashed circles in Figure 3 (a)). The recovery demonstrates that subjecting the solutions to LAOS will not result in complete permanent deformation.

Figure 3 (b) is the calculated permanent deformation for $\mathrm{H} 0-\mathrm{H} 3$ and $\mathrm{P} 0-\mathrm{P} 3$. The data revealed that the addition of caffeine decreased the recoverable deformation for both biopolymers. For the PUL solutions, the addition of $3 \% \mathrm{w} / \mathrm{w}$ caffeine decreased the recoverable deformation by $23.9 \%$, from $64.1 \%$ to $40.2 \%$. A further $16.4 \%$ decrease was observed when the caffeine content was increased to $10 \%$ w/w. For the HPMC samples, the addition of $3 \% \mathrm{w} / \mathrm{w}$ caffeine decreased the recoverable deformation by $15.3 \%$, from $70.3 \%$ to $55.0 \%$; and a further $24.5 \%$ reduction when the caffeine content was increased to $10 \% \mathrm{w} / \mathrm{w}$.

\subsection{PAM Printed Film Properties}

PAM was successfully utilized to print caffeine-loaded PUL, HPMC and PUL-HPMC hybrid films. The printing parameters were found to vary depending on the formulation. P0 and H0 were printable at $65 \mathrm{kPa}, \mathrm{P} 1$ and $\mathrm{H} 1$ were printable at $80 \mathrm{kPa}$, which was an increase in pressure by 23 $\%$. P2 and H2 required 196 and $140 \mathrm{kPa}$, which amounted to increases of $145 \%$ and $75 \%$, respectively, a considerable increase compared with other formulations. The addition of water reduced the required printing pressures to 60 and $40 \mathrm{kPa}$ for $\mathrm{P} 3 / \mathrm{H} 3$ and $\mathrm{P} 4 / \mathrm{H} 4$, respectively. Thus, increasing the water from 60 to $66 \% \mathrm{w} / \mathrm{w}$ for films loaded with $10 \% \mathrm{w} / \mathrm{w}$ caffeine, whilst decreasing the biopolymer content from 23 to $17 \% \mathrm{w} / \mathrm{w}$, had a marked effect on the extrusion pressure. Despite the increase in water content, exaggerated spreading of the solutions was not observed, with the films maintaining their $30 \times 10 \mathrm{~mm}$ rectangular structure. The thicknesses of the films determined by both a Vernier caliper and SEM ranged between 100 to $150 \mu \mathrm{m}$. Examples 
of PAM printed films are presented in Figure 4. A notable distinction was that caffeine-free films tended to curl (Figure 4 (a)). Given that both P4 and H4 required the least pressure to print whilst maintaining print quality, both these formulations were selected for hybrid film fabrication.

The PUL-HPMC (50/50) blend solution was also processed through the 3D printer. The blended solution was found to be extrudable at $40 \mathrm{kPa}$, however once dried it yielded disjointed films with visually noticeable voids, as portrayed in Figure 5. Furthermore, the films were also visually observed to possess a rough, fibrous surface compared with the single biopolymer solutions. Increasing the pressure reduced the level of voids present within the films, and at $80 \mathrm{kPa}$ no voids were ostensibly present (Figure 5 (e)). Hence, a two-fold increase in pressure was needed to fabricate PUL-HPMC (50/50) blended solutions compared with H4 and P4 solutions, which had similar biopolymer, drug, plasticizer and water proportions. Moreover, the fibrous surface was maintained at $80 \mathrm{kPa}$.

However, the films could not be removed from the petri dish without fracturing, as depicted in Figure 5 (f). Hence, no mechanical tests could be performed on PUL-HPMC (50/50) blend films. Chemical structural analyses using XRD and FTIR were performed on the PUL-HPMC (50/50) films (Figure S5 and S6), to determine whether phase separation had occurred during the printing process. The results revealed no phase separation, and that films were comprised of PUL, HPMC and caffeine. A detailed description of the XRD and FTIR results can be found in the supplementary document.

\subsection{Morphological Analysis}

The surface morphology of the printed films was investigated using SEM and presented in Figure 6. The caffeine loaded PUL and HPLC films were prepared and structured through the alignment of fibers by printing to create a mesh structure. In all of the films, the drug-loaded polymers formed web-like structures between the fibers. A complete fiber formation with a smooth surface was obtained in the case of caffeine loaded HPMC films $(\mathrm{H} 2)$, whereas rough fiber surfaces were seen for caffeine loaded PUL films (P2). The fibrous structure could be seen for the HPMC-PUL 50/50 blend films as well although the fibers interweaved with each other along with lots of defects on 
the surface of the film. This presumably reflects the incompatible nature of the HPMC-PUL 50/50 composition, which resulted in the inhomogeneous fibers in the structure of the films.

\subsection{Tensile Mechanical Properties}

As-dried films were analysed for their tensile properties by examining the ultimate tensile strength (UTS), elastic modulus and elongation break. The results are displayed in Figure 7 and Figure 8 for PUL and HPMC, respectively. For the PUL films, it was determined that the inclusion of caffeine reduced their UTS (Figure 7 (a)). From the results, it was clear that the addition of caffeine influenced the mechanical properties of PUL, wherein an increase in Elastic modulus was observed, whilst both the UTS and elongation break decreased. Hybrid printing of P4 revealed no significant difference in mechanical properties to that of the single-mode printing. A similar trend was observed with the HPMC films with the addition of caffeine, albeit only a marginal increase in the elastic modulus was observed. Again, the hybrid printing displayed similar mechanical properties regarding the orientation of the print, except for the elastic moduli of the transverse H4

films, which were found to be noticeably lower than those of the longitudinal films. Overall, the results revealed that dual printing of PUL and HPMC had no significant effect on the mechanical properties.

Once dual-printing was accomplished for PUL-PUL and HPMC-HPMC films, PULLHPMC films were investigated. Printing PUL with HPMC longitudinally (PUL-HPMC-L) had a marked effect on the tensile properties, as depicted in Figure 9. The mean UTS increased from 8.90 MPa for P4 to 14.50 MPa when composited, which was an increase of $61 \%$, with no overlap in data observed. The mean elastic modulus increased from 1.17 to $1.56 \mathrm{GPa}$ when printed with $\mathrm{HPMC}$, an increase of $33 \%$; with the maximum for P4 determined as $1.27 \mathrm{GPa}$, and the minimum for the longitudinal film calculated as $1.29 \mathrm{GPa}$, there was no overlap in values. The mean elongation break for pullulan-only films was $1.48 \%$, which increased to $1.77 \%$ when dual printed. There was an overlap in results between the pure PUL and dual printed PUL, however, in contrast to the UTS and elastic modulus, the HPMC films did not exhibit the greater values of the two polymer films. 
The transverse-oriented films (PUL-HPMC-T) did not have the marked improvement observed with the longitudinal films. The mean UTS was $9.42 \mathrm{MPa}$, a marginal improvement to that of P4. In contrast, the elastic modulus and elongation break, $1.08 \mathrm{GPa}$ and $1.21 \%$, respectively, were noticeably lower than P4. Therefore, the results demonstrate that longitudinal orientation yielded optimal results for PUL-HPMC composites.

\subsection{Disintegration Test}

The disintegration times of the dual-printed hybrid film comprised of $\mathrm{H} 4$ and $\mathrm{P} 4$ were recorded and the results listed in Table 2. The results for the individual films are also included for comparison. It was discovered that the time to disintegration for the hybrid films was $35.4 \mathrm{~s}$, which was between the time it took for P4 and H4 films to disintegrate (27.8 and $45.2 \mathrm{~s}$, respectively). The results infer that the resultant disintegration time of the hybrid films was an amalgamation of the individual PUL and HPMC films.

\section{Discussion}

The results of the study demonstrate that PAM is a facile, cost-effective and rapid alternative for hybrid film fabrication. The salient finding of this study was that PUL can be blended with HPMC to produce a viable film when processed via PAM, where an increase in mean tensile properties of PUL was observed. This is in contrast to previous work that reported a decrease in film mechanical properties of PUL-HPMC blends processed via solution casting (Prasad et al., 2008). Furthermore, the printing orientation of PUL-HPMC was found to affect the tensile properties. Thus, in addition to the inherent properties of the two polymers, the printing orientation can be exploited to tailor the mechanical properties. Currently, there are no quantifiable guidelines for oral film tensile properties (Borges et al., 2015b). Preis et al. (2014) reported the elongation break of commerciallyavailable products, in which the values were between $1.03 \pm 0.21$ to $6.54 \pm 0.89 \%$ (Preis et al., 2014), which are similar to the results obtained herein.

As mentioned, 3DP can create complex structures. Considering that pediatric patients are often targeted recipients of OFs, the ability to produce different and enticing shapes can make the medication more appealing (Norman et al., 2017). For previous technologies such as injection 
molding and extrusion, different shapes require costly molds or dies. Moreover, traditional fabrication techniques are limited in their capacity for personalized, on-demand delivery, where demographics such as paediatrics and geriatrics require markedly different dosing (Trenfield et al., 2019). However, recent clinical work has demonstrated that 3DP can achieve this goal (Goyanes et al., 2019). Therefore, 3DP has the potential to meet a clinical need for on-demand OF fabrication. Previous work has used fused deposition modelling (FDM), a commonly-used 3DP technology, to fabricate films (Jamróz et al., 2017). The study reported film thicknesses of $197 \mu \mathrm{m}$, which is larger than the $100 \mu \mathrm{m}$ fabricated herein (Ehtezazi et al., 2018; Jamróz et al., 2017). Moreover, PAM does not require expensive instruments to generate the feedstock, whereas FDM requires hot-melt extruders. A key advantage of PAM over most 3DP technologies is that it can process a wide variety of polymers, whereas FDM is limited to thermoplastics. The film thicknesses were comparable with those reported by inkjet printing (IJP), a 3DP technology widely used for OFs, although lower thicknesses can be achieved by IJP (Dodoo et al., 2020; Eleftheriadis et al., 2020; Öblom et al., 2020). However, IJP is limited with regards to drug loading, whereas PAM can achieve loading as high as $80 \%$ w/w (Keikhosravi et al., 2020; Khaled et al., 2018). Further work will be needed to realize the full potential of PAM for the fabrication of OFs.

\section{Conclusion}

The present study elucidated several key findings pertaining to the hybrid printing of PUL and HPMC, which are inherently immiscible when mixed at 50-50\% w/w. It was found that adding HPMC in dual printing increased the mean strength of the pullulan films, from 8.9 to $14.5 \mathrm{MPa}$ and 9.4 MPa for longitudinal and transverse, respectively. The mean elastic modulus also increased from 1.17 to $1.56 \mathrm{GPa}$ when dual printed with HPMC in the longitudinal direction but decreased to $1.08 \mathrm{GPa}$ with the transverse orientation. The mean elongation break was found to increase with the longitudinal orientation, from $1.48 \%$ to $1.77 \%$, but a decrease was observed with the transverse orientation to $1.21 \%$. Hence, it was determined that higher mechanical properties of composite films were favored by the longitudinal orientation. 


\section{Acknowledgments}

ME would like to acknowledge the Kempe Foundation for supporting this work (Grant no.: SMK-1640). DN is grateful for the financial support provided by Åforsk (ref.nr 18-459)

\section{References}

Awad, A., Fina, F., Goyanes, A., Gaisford, S., Basit, A.W., 2020a. 3D printing: Principles and pharmaceutical applications of selective laser sintering. Int. J. Pharm. 586, 119594. https://doi.org/https://doi.org/10.1016/j.ijpharm.2020.119594

Awad, A., Yao, A., Trenfield, S.J., Goyanes, A., Gaisford, S., Basit, A.W., 2020b. 3D Printed Tablets (Printlets) with Braille and Moon Patterns for Visually Impaired Patients. Pharmaceutics 12, 172.

Borges, A.F., Silva, C., Coelho, J.F.J., Simões, S., 2015a. Oral films: Current status and future perspectives II - Intellectual property, technologies and market needs. J. Control. Release 206, 108-121. https://doi.org/https://doi.org/10.1016/j.jconrel.2015.03.012

Borges, A.F., Silva, C., Coelho, J.F.J., Simões, S., 2015b. Oral films: Current status and future perspectives: I - Galenical development and quality attributes. J. Control. Release 206, 119. https://doi.org/https://doi.org/10.1016/j.jconrel.2015.03.006

Cristescu, R., Stamatin, I., Mihaiescu, D.E., Ghica, C., Albulescu, M., Mihailescu, I.N., Chrisey, D.B., 2004. Pulsed laser deposition of biocompatible polymers: a comparative study in case of pullulan. Thin Solid Films 453-454, 262-268. https://doi.org/https://doi.org/10.1016/j.tsf.2003.11.145

Dodoo, C.C., Stapleton, P., Basit, A.W., Gaisford, S., 2020. The potential of Streptococcus salivarius oral films in the management of dental caries: An inkjet printing approach. Int. J. Pharm. 591, 119962. https://doi.org/https://doi.org/10.1016/j.ijpharm.2020.119962

Ehtezazi, T., Algellay, M., Islam, Y., Roberts, M., Dempster, N.M., Sarker, S.D., 2018. The Application of 3D Printing in the Formulation of Multilayered Fast Dissolving Oral Films. J. Pharm. Sci. 107, 1076-1085. https://doi.org/10.1016/j.xphs.2017.11.019

Elbadawi, M., 2019. Rheological and Mechanical Investigation into the Effect of Different Molecular Weight Poly(ethylene glycol)s on Polycaprolactone-Ciprofloxacin Filaments. ACS Omega 4, 5412-5423. https://doi.org/10.1021/acsomega.8b03057

Elbadawi, M., 2018. Polymeric Additive Manufacturing: The Necessity and Utility of Rheology, in: Rivera-Armenta, J.L., Cruz, B.A.S. (Eds.), Polymer Rheology. IntechOpen, Rijeka. https://doi.org/10.5772/intechopen.77074

Elbadawi, M., Ong, J.J., Pollard, T.D., Gaisford, S., Basit, A.W., 2020. Additive Manufacturable Materials for Electrochemical Biosensor Electrodes. Adv. Funct. Mater. n/a, 2006407. https://doi.org/https://doi.org/10.1002/adfm.202006407 
Eleftheriadis, G., Monou, P.K., Andriotis, E., Mitsouli, E., Moutafidou, N., Markopoulou, C., Bouropoulos, N., Fatouros, D., 2020. Development and Characterization of Inkjet Printed Edible Films for Buccal Delivery of B-Complex Vitamins. Pharmaceuticals 13, 203.

Goyanes, A., Buanz, A.B.M., Hatton, G.B., Gaisford, S., Basit, A.W., 2015. 3D printing of modified-release aminosalicylate (4-ASA and 5-ASA) tablets. Eur. J. Pharm. Biopharm. 89, 157-162. https://doi.org/https://doi.org/10.1016/j.ejpb.2014.12.003

Goyanes, A., Madla, C.M., Umerji, A., Duran Piñeiro, G., Giraldez Montero, J.M., Lamas Diaz, M.J., Gonzalez Barcia, M., Taherali, F., Sánchez-Pintos, P., Couce, M.-L., Gaisford, S., Basit, A.W., 2019. Automated therapy preparation of isoleucine formulations using 3D printing for the treatment of MSUD: First single-centre, prospective, crossover study in patients. Int. J. Pharm. 567, 118497. https://doi.org/https://doi.org/10.1016/j.ijpharm.2019.118497

Hadal, R.S., Misra, R.D.K., 2005. Scratch deformation behavior of thermoplastic materials with significant differences in ductility. Mater. Sci. Eng. A 398, 252-261. https://doi.org/https://doi.org/10.1016/j.msea.2005.03.028

Jamróz, W., Kurek, M., Łyszczarz, E., Szafraniec, J., Knapik-Kowalczuk, J., Syrek, K., Paluch, M., Jachowicz, R., 2017. 3D printed orodispersible films with Aripiprazole. Int. J. Pharm. 533, 413-420. https://doi.org/https://doi.org/10.1016/j.ijpharm.2017.05.052

Kanmani, P., Lim, S.T., 2013. Development and characterization of novel probiotic-residing pullulan/starch edible films. Food Chem. 141, 1041-1049. https://doi.org/https://doi.org/10.1016/j.foodchem.2013.03.103

Karki, S., Kim, H., Na, S.-J., Shin, D., Jo, K., Lee, J., 2016a. Thin films as an emerging platform for drug delivery. Asian J. Pharm. Sci. 11, 559-574. https://doi.org/https://doi.org/10.1016/j.ajps.2016.05.004

Karki, S., Kim, H., Na, S.-J., Shin, D., Jo, K., Lee, J., 2016b. Thin films as an emerging platform for drug delivery. Asian J. Pharm. Sci. 11, 559-574. https://doi.org/https://doi.org/10.1016/j.ajps.2016.05.004

Keikhosravi, N., Mirdamadian, S.Z., Varshosaz, J., Taheri, A., 2020. Preparation and characterization of polypills containing aspirin and simvastatin using 3D printing technology for the prevention of cardiovascular diseases. Drug Dev. Ind. Pharm. 46, 1665-1675. https://doi.org/10.1080/03639045.2020.1820034

Khaled, S.A., Alexander, M.R., Wildman, R.D., Wallace, M.J., Sharpe, S., Yoo, J., Roberts, C.J., 2018. 3D extrusion printing of high drug loading immediate release paracetamol tablets. Int. J. Pharm. 538, 223-230. https://doi.org/https://doi.org/10.1016/j.ijpharm.2018.01.024

Mazumder, S., Pavurala, N., Manda, P., Xu, X., Cruz, C.N., Krishnaiah, Y.S.R., 2017. Quality by Design approach for studying the impact of formulation and process variables on product quality of oral disintegrating films. Int. J. Pharm. 527, 151-160. https://doi.org/https://doi.org/10.1016/j.ijpharm.2017.05.048

McKeen, L.W., 2017. 13 - Environmentally Friendly Polymers, in: McKeen, L.W.B.T.-P.P. of P. and E. (Fourth E. (Ed.), Plastics Design Library. William Andrew Publishing, pp. 305-323. 
https://doi.org/https://doi.org/10.1016/B978-0-323-50859-9.00013-0

Norman, J., Madurawe, R.D., Moore, C.M. V, Khan, M.A., Khairuzzaman, A., 2017. A new chapter in pharmaceutical manufacturing: 3D-printed drug products. Adv. Drug Deliv. Rev. 108, 39-50. https://doi.org/https://doi.org/10.1016/j.addr.2016.03.001

Öblom, H., Cornett, C., Bøtker, J., Frokjaer, S., Hansen, H., Rades, T., Rantanen, J., Genina, N., 2020. Data-enriched edible pharmaceuticals (DEEP) of medical cannabis by inkjet printing. Int. J. Pharm. 589, 119866. https://doi.org/https://doi.org/10.1016/j.ijpharm.2020.119866

Prajapati, V.D., Chaudhari, A.M., Gandhi, A.K., Maheriya, P., 2018. Pullulan based oral thin film formulation of zolmitriptan: Development and optimization using factorial design. Int. J. Biol. Macromol. 107, 2075-2085. https://doi.org/https://doi.org/10.1016/j.ijbiomac.2017.10.082

Prajapati, V.D., Jani, G.K., Khanda, S.M., 2013. Pullulan: An exopolysaccharide and its various applications. Carbohydr. Polym. 540-549. https://doi.org/https://doi.org/10.1016/j.carbpol.2013.02.082

Prasad, P., Guru, G.S., Shivakumar, H.R., Rai, K.S., 2008. Miscibility, thermal, and mechanical studies of hydroxypropyl methylcellulose/pullulan blends. J. Appl. Polym. Sci. 110, 444-452. https://doi.org/10.1002/app.28575

Preis, M., Knop, K., Breitkreutz, J., 2014. Mechanical strength test for orodispersible and buccal films. Int. J. Pharm. 461, 22-29. https://doi.org/https://doi.org/10.1016/j.ijpharm.2013.11.033

Preis, M., Woertz, C., Kleinebudde, P., Breitkreutz, J., 2013. Oromucosal film preparations: classification and characterization methods. Expert Opin. Drug Deliv. 10, 1303-1317. https://doi.org/10.1517/17425247.2013.804058

Robles-Martinez, P., Xu, X., Trenfield, S.J., Awad, A., Goyanes, A., Telford, R., Basit, A.W., Gaisford, S., 2019. 3D printing of a multi-layered polypill containing six drugs using a novel stereolithographic method. Pharmaceutics 11, 274.

Sathya, A.B., Sivashankar, R., Kanimozhi, J., Thirunavukkarasu, A., Santhiagu, A., Sivasubramanian, V., 2018. Biodegradable Plastics for a Green and Sustainable Environment, in: Sivasubramanian, V. (Ed.), Bioprocess Engineering for a Green Environment.

Scarpa, M., Stegemann, S., Hsiao, W.-K., Pichler, H., Gaisford, S., Bresciani, M., Paudel, A., Orlu, M., 2017. Orodispersible films: Towards drug delivery in special populations. Int. J. Pharm. 523, 327-335. https://doi.org/https://doi.org/10.1016/j.ijpharm.2017.03.018

Shingel, K.I., 2004. Current knowledge on biosynthesis, biological activity, and chemical modification of the exopolysaccharide, pullulan. Carbohydr. Res. 339, 447-460. https://doi.org/https://doi.org/10.1016/j.carres.2003.10.034

Silva, B.M.A., Borges, A.F., Silva, C., Coelho, J.F.J., Simões, S., 2015. Mucoadhesive oral films: The potential for unmet needs. Int. J. Pharm. 494, 537-551. https://doi.org/https://doi.org/10.1016/j.ijpharm.2015.08.038

Slavkova, M., Breitkreutz, J., 2015. Orodispersible drug formulations for children and elderly. Eur. 
J. Pharm. Sci. 75, 2-9. https://doi.org/https://doi.org/10.1016/j.ejps.2015.02.015

Sugumaran, K.R., Ponnusami, V., 2017. Review on production, downstream processing and characterization of microbial pullulan. Carbohydr. Polym. 173, 573-591. https://doi.org/https://doi.org/10.1016/j.carbpol.2017.06.022

Tabasum, S., Noreen, A., Maqsood, M.F., Umar, H., Akram, N., Nazli, Z.-H., Chatha, S.A.S., Zia, K.M., 2018a. A review on versatile applications of blends and composites of pullulan with natural and synthetic polymers. Int. J. Biol. Macromol. 120, 603-632. https://doi.org/https://doi.org/10.1016/j.ijbiomac.2018.07.154

Tabasum, S., Noreen, A., Maqsood, M.F., Umar, H., Akram, N., Nazli, Z.-H., Chatha, S.A.S., Zia, K.M., 2018b. A review on versatile applications of blends and composites of pullulan with natural and synthetic polymers. Int. J. Biol. Macromol. 120, 603-632. https://doi.org/https://doi.org/10.1016/j.ijbiomac.2018.07.154

Trenfield, S.J., Awad, A., Madla, C.M., Hatton, G.B., Firth, J., Goyanes, A., Gaisford, S., Basit, A.W., 2019. Shaping the future: recent advances of 3D printing in drug delivery and healthcare. Expert Opin. Drug Deliv. 16, 1081-1094. https://doi.org/10.1080/17425247.2019.1660318

Trenfield, S.J., Tan, H.X., Goyanes, A., Wilsdon, D., Rowland, M., Gaisford, S., Basit, A.W., 2020. Non-destructive dose verification of two drugs within 3D printed polyprintlets. Int. J. Pharm. 577, 119066. https://doi.org/https://doi.org/10.1016/j.ijpharm.2020.119066

Vivero-Lopez, M., Xu, X., Muras, A., Otero, A., Concheiro, A., Gaisford, S., Basit, A.W., AlvarezLorenzo, C., Goyanes, A., 2021. Anti-biofilm multi drug-loaded 3D printed hearing aids. Mater. Sci. Eng. C 119, 111606. https://doi.org/https://doi.org/10.1016/j.msec.2020.111606

Vuddanda, P.R., Montenegro-Nicolini, M., Morales, J.O., Velaga, S., 2017. Effect of plasticizers on the physico-mechanical properties of pullulan based pharmaceutical oral films. Eur. J. Pharm. Sci. 96, 290-298. https://doi.org/https://doi.org/10.1016/j.ejps.2016.09.011

Xu, X., Awad, A., Martinez, P.R., Gaisford, S., Goyanes, A., Basit, A.W., 2020. Vat photopolymerization 3D printing for advanced drug delivery and medical device applications. J. Control. Release. https://doi.org/https://doi.org/10.1016/j.jconrel.2020.10.008

Xu, X., Goyanes, A., Trenfield, S.J., Diaz-Gomez, L., Alvarez-Lorenzo, C., Gaisford, S., Basit, A.W., 2021. Stereolithography (SLA) 3D printing of a bladder device for intravesical drug

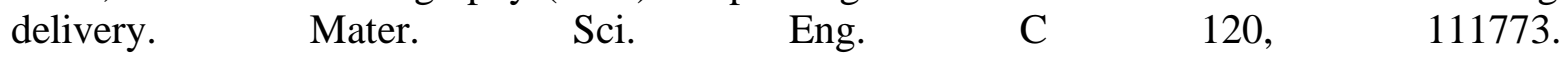
https://doi.org/https://doi.org/10.1016/j.msec.2020.111773 


\section{Figures}

Figure 1 Schematic representation of the fabrication process.

Figure 2. Viscosity plots as a function of shear rate for (a) PUL, (b) HPMC and (c) a PUL-HPMC $(50 / 50)$ blend solutions. ( $n \geq 3$; error bars represent the standard deviation).

Figure 3. (a)A representative plot illustrating the effect of LAOS on the storage modulus. An initial small amplitude (1\% strain for 45 seconds) was applied, followed by a LAOS (1000 \% for 45 seconds) to replicate the effect of shearing during extrusion, which in turn was followed by a SAOS bout to observe the recovery behavior of the solutions with regards to its elastic facet (dashed circles - a continuous time sweep at $1 \% ; n=\geq 3$ ). (b) The effect of caffeine on the ability of PUL and HPMC solutions to recover. ( $\mathrm{n} \geq 3$; ' \pm ' represents the standard deviation).

Figure 4. Examples of films fabricated via PAM. (a) PUL sample without caffeine; (b) HPMC sample with $10 \% \mathrm{w} / \mathrm{w}$ caffeine; (c) a PUL-HPMC-L hybrid film printed in the longitudinal orientation (d) a PUL-HPMC-T hybrid film printed in the transverse orientation.

Figure 5. Photographic images illustrating films fabricated from a PUL-HPMC (50/50) blend formulation. (ruler hash marks are in $\mathrm{mm}$ )

Figure 6. SEM top section images depicting the surface morphology of the printed films for (a,b) Caffeine loaded HPMC (H2); (c,d) Caffeine loaded PUL (P2); and (e,f) for PUL-HPMC 50/50 blend films.

Figure 7. Tensile properties of the PUL films presented as box plots, which delineate the mean, median, first and third quartile, and the range. (a) are the ultimate tensile strength, (b) are the elastic moduli and (c) the elongation break. (Long - longitudinal and Trans- Transverse orientations; $\mathrm{n} \geq$ $6)$.

Figure 8. Tensile properties of the HPMC films presented as box plots, which delineate the mean, median, first and third quartile, the range and outliers. (a) are the ultimate tensile strength, (b) are the elastic moduli and (c) the elongation break. (Long - longitudinal and Trans- Transverse orientations; $n \geq 6$ ).

Figure 9. PUL-HPMC dual printed tensile data with the (a) ultimate tensile strength, (b) elastic modulus and (c) elongation break results presented as box plots. The polymer-only data of the respective polymers are included for comparison. $(n \geq 6)$.

\section{Tables}

Table 1. The compositions (\% $/ \mathrm{w})$ of the formulations/printed films with their corresponding sample code (P0-P4 stand for PUL formulations, H0-H4 stand for HPMC formulations, PULHPMC blend stand for 50/50 blend mixtures).

Table 2. Disintegration time for the dual-printed hybrid film comprised of H4 and P4 films. The results for the individual films are also included for comparison. $(n=6)$. 